\title{
Parameter Identification Of Time-Delay Systems: A Flatness Based Approach *
}

\author{
René Schenkendorf* Udo Reichl ${ }^{*, * *}$ Michael Mangold* \\ * Max Planck Institute for Dynamics of Complex Technical Systems, \\ 39106 Magdeburg, Sandtorstraße 1 - Germany \\ (e-mail: rschenke@mpi-magdeburg.mpg.de). \\ ** Bioprocess Engineering, Otto von Guericke University, \\ 39106 Magdeburg, Universitätsplatz 2 - Germany
}

\begin{abstract}
The use of mathematical models is widely established in various fields of application. To name but a few of their major applications, mathematical models can improve the controller design of complex technical systems or are able to facilitate the understanding of highly complex biochemical systems. No matter what mathematical models are used for, however, they fail to perform the intended task if they are badly parameterized.

In general, during the process of parameterization one tries to make differences between simulation results and measurement data as small as possible. Under the assumption of a suitable model candidate this is done by choosing optimal model parameters. Unfortunately, the majority of used models cannot be solved analytically. For example, many dynamical processes are described by systems of ordinary differential equations (ODEs). Usually, analytical solutions do not exist. Although quite efficient numerical routines are available they usually slow down the parameterization process dramatically.

The situation is even more demanding if one has to deal with processes that are described by delay differential equations (DDEs). Commonly, standard DDE solvers show a lack of efficiency as well as of robustness, i.e., they are likely to fail to solve the underlying DDE system.

Consequently, it would be of great benefit to eliminate any need of numerical ODE/DDE solvers. Here, the concept of flat inputs comes into play. The key aspect is to transform the DDE system into an algebraic input/output representation, i.e., the inputs of the system are expressed analytically by the outputs and derivatives thereof. Now, the objective of parameterization is to minimize differences between these flat inputs and the physical inputs of the related process. As no numerical DDE solver is involved there is a significant speedup of the parameter identification step. In addition, the presented approach is closely linked to optimal experimental design for parameter identification. In particular, the reformulation of the cost function also affects parameter sensitivities. Using the same measurement data it is possible that previously insensitive model parameters become sensitive. To check this, global parameter sensitivities are determined by Sobol' Indices of first order.

All results are demonstrated for the example of a mathematical model of the influenza A virus production.
\end{abstract}

Keywords: Delay Differential Equations, Parameter Identification, Parameter Sensitivities, Flat Inputs

\section{INTRODUCTION}

Essentials in systems biology can be described and analyzed using deterministic models. For this purpose expert knowledge has to be converted in a suitable model structure determining in which manner model components interact. Frequently, such an attempt ends in ordinary differential equations (ODEs). Often, the underlaying principles of biochemical processes are only vaguely known and not directly accessible to measurement data. Therefore, unknown or unaccessible subprocesses are approximated by time-delay processes. Instead of ODEs these sys- tems are described by delay differential equations (DDEs). Here, additional time delay parameters $\tau$ influence the input/output behavior of the mathematical model. Especially, in the field of biology one has to accomplish the feat to determine a vast number of unknowns from sparse data, i.e., only a subset of quantities can be measured directly at a limited number of time points $t_{k}$. Under these adverse conditions, the identification of the model parameters $\theta$ as well as time delay parameters $\tau$ is a challenge.

In the following, a novel framework of the identification of $\theta$ and $\tau$ is discussed for the example of a mathematical model of the influenza A virus production.

\footnotetext{
* Financial support from the German Federal Ministry of Education and Research (BMBF) under Grant 0315505B is gratefully acknowledged.
} 


\section{BACKGROUND}

\subsection{Flat Inputs for Parameter Identification}

Dynamical processes can be described by deterministic differential equation models. After deriving a suitable model structure, unknown model parameters $\theta \in \mathbb{R}^{p}$ have to be identified by measurement data $y^{\text {data }}(t) \in \mathbb{R}^{m}$ to obtain a predictive model. Here, the class of delay differential equations is the focus of the presented work.

Considering a DDE system (Eq. 1-3) one tries to minimize the differences between simulation results $y^{\operatorname{sim}}(t)$ and measurement data $y^{\text {data }}(t)$ during the step of parameter identification.

$$
\begin{array}{rlrl}
\dot{x}(t) & =f(x(t), x(t-\tau), \theta, u(t)) & ; x \in \mathbb{R}^{n}, u \in \mathbb{R}^{l} \\
y^{s i m}(t) & =h(x(t), x(t-\tau), \theta, u(t)) & & ; y^{s i m} \in \mathbb{R}^{m} \\
x(t) & =\Xi(t), \quad t \in[-\tau, 0] & &
\end{array}
$$

For this purpose, a suitable cost function (Eq. 4) taking these differences into account has to be evaluated by a numerical optimization routine.

$$
\arg \min _{\theta, \tau} J_{y}(\theta, \tau)=\sum_{k=1}^{K}\left(y^{\text {data }}\left(t_{k}\right)-y^{s i m}\left(t_{k}\right)\right)^{2}
$$

One limiting factor in applying this approach is the repeated need of numerical integration of the underlying DDEs (Eq. 1). Efficient and robust DDE-solver are hard to find and if at all possible, the overall parameter identification ends in a very cpu-intensive process. In addition, to solve DDEs, related initial functions $\Xi(t), t \in[-\tau, 0]$ have to be known explicitly. Consequently, it would be of great benefit to eliminate the numerical integration step. Here, the concept of flat inputs [Waldherr and Zeitz (2008)] comes into play. The main idea is to transform the DDE system (Eq. 1-3) into an algebraic input/output representation, i.e., the flat inputs $u^{\text {flat }}(t)$ (Eq. 5) of the system can be expressed explicitly by the outputs (Eq. 2) and their derivatives.

$$
u^{\text {flat }}(t)=\Omega\left(y(t), \dot{y}(t), \ldots, y^{(\beta)}(t), \tau, \theta\right)
$$

In detail, one attempts to determine input configurations in such a way that the measured outputs $y^{\operatorname{sim}}(t)$ are flat outputs, i.e., steering the related process via flat inputs a differentially flat system is obtained. Basics about the concept of differential flatness can be found in control theory literature [Sira-Ramirez and Agrawal (2004); Graichen et al. (2005); Lévine (2009)]. Here, one should stress that flat inputs $u^{\text {flat }}(t)$ do not have to correspond to real physical inputs $u^{\text {real }}(t)$ of the considered process. In this case, flat inputs may be accepted as fictitious inputs. Furthermore, systems can exist that lack any kind of flat inputs, i.e., absolutely no possible input configuration ensures the property of differential flatness. The problem of determining flat inputs is addressed in subsection 2.2 separately.
For the purpose of parameter identification flat inputs can be used to define novel cost functions. If flat inputs have real physical counterparts $u^{\text {real }}(t)$, such a cost function measures differences between $u^{\text {real }}(t)$ and $u^{\text {flat }}(t)$. One possible cost function is given below (Eq. 6).

$$
\arg \min _{\theta, \tau} J_{u}(\theta, \tau)=\int_{0}^{T}\left(u^{r e a l}(t)-u^{\text {flat }}(t)\right)^{2} d t
$$

In the case that flat inputs are only fictitious, i.e., real physical counterparts do not exist, the following cost function (Eq. 7) has to be implemented.

$$
\arg \min _{\theta, \tau} J_{u}(\theta, \tau)=\int_{0}^{T}\left(u^{\text {flat }}(t)\right)^{2} d t
$$

If needed time derivatives of output functions in (Eq. 5) do not exist at certain time points, these time intervals have to be excluded in (Eq. 6) and (Eq. 7), respectively.

A much more serious problem in the framework of flat inputs for parameter identification are measurement imperfections. In practice, real measurement data $y^{\text {data }}\left(t_{k}\right)$ are only available at discrete time points and corrupted by measurement noise. Here, the concept of functional data analysis [Ramsay and Silverman (2005); Poyton et al. (2006); Varziri et al. (2008)] provides sophisticated methods to determine surrogate output functions (Eq. 8) that can be incorporated in (Eq. 5).

$$
y^{\text {surr }}(t)=c^{T} \Phi(t)
$$

In what follows, B-splines [Ramsay and Silverman (2005)] are chosen as basis functions $\Phi(t)$ that have to be fitted to measurement data $y^{\text {data }}\left(t_{k}\right)$ by optimally chosen coefficients $c$ (Eq. 8). Both optimization problems can be solved simultaneously leading to the final cost function (Eq. 9).

$$
\begin{aligned}
\arg \min _{c, \theta, \tau} J_{u}(c, \theta, \tau)= & \sum_{k=1}^{K}\left(y^{\text {data }}\left(t_{k}\right)-y^{\text {surr }}\left(t_{k}\right)\right)^{2}+ \\
& \int_{0}^{T}\left(u^{\text {real }}(t)-u^{\text {flat }}(t)\right)^{2} d t
\end{aligned}
$$

Again, if real physical input counterparts $u^{\text {real }}(t)$ are missing but fictitious flat inputs $u^{\text {flat }}(t)$ exist, the cost function (Eq. 9) is simplified to (Eq. 10).

$$
\begin{array}{r}
\arg \min _{c, \theta, \tau} J_{u}(c, \theta, \tau)=\sum_{k=1}^{K}\left(y^{\text {data }}\left(t_{k}\right)-y^{\text {surr }}\left(t_{k}\right)\right)^{2}+ \\
\int_{0}^{T}\left(u^{\text {flat }}(t)\right)^{2} d t
\end{array}
$$




\subsection{Determination of Flat Inputs}

Here, some helpful hints about the determination of flat inputs $u^{\text {flat }}(t)$ are given. In fact, a necessary and sufficient condition for the existence of a flat input is only available for ODE systems with single input and single output [Waldherr and Zeitz (2008)]. For the more complex case of multiple inputs and multiple outputs no conditions at all are on hand currently [Waldherr and Zeitz (2010)]. The same is true for DDE systems, i.e., there is no rigorous proof of the existence of flat inputs in this case.

Nevertheless, a heuristic approach is shortly presented in the following. In flat system theory it is well known that the dimensions of the input $u \in \mathbb{R}^{l}$ have to be equivalent to the dimensions of the output $y \in \mathbb{R}^{m}, l \stackrel{!}{=} m$. For a given number of measurable outputs the number of needed flat inputs $u_{i}^{\text {flat }}(t)$ is defined automatically.

Now, the question at which states $x_{i}(t)$ these flat inputs have to act on has to be addressed. In principle, this can be achieved heuristically by the framework of structural analysis. First considerations in this direction can be found in the context of flat outputs [Wey (2002)]. The structural analysis is based on directed graphs (digraphs) $D(v, e)$ with $n$ different nodes $v_{i}$ representing the states of the DDE system (Eq. 1). The existence of an edge $e_{i, j}$ from node $v_{i}$ to $v_{j}$ is determined by non-zero elements $a_{j, i}$ of the adjacency matrix $A^{*}$. The $a_{i, j}$ element of $A^{*}$ is set to 1 if derivatives $\frac{\partial f_{i}(x(t), x(t-\tau), \theta)}{\partial x_{j}(t)}$ or $\frac{\partial f_{i}(x(t), x(t-\tau), \theta)}{\partial x_{j}(t-\tau)}$ exist and to 0 if this is not the case. In a similar way, an adjacency matrix $C^{*}$ of the output functions $y^{\text {sim }}(t)$ (Eq. 2) can be derived.

Flat inputs $u^{\text {flat }}(t)$ should act on those nodes $v_{i}$ that are the most distant nodes related to the outputs $y^{\text {sim }}(t)$, i.e., there is a maximum number of edges to get from $u^{\text {flat }}(t)$ to $y^{\text {flat }}(t)$ traveling along the shortest paths.

In order to illustrate the presented flat input selection strategy, a parameter-free DDE system (Eq. 11) is considered.

$$
\begin{aligned}
\dot{x}_{1}(t) & =x_{3}\left(t-\tau_{1}\right) \\
\dot{x}_{2}(t) & =x_{1}(t) \\
\dot{x}_{3}(t) & =x_{2}(t) \\
y^{s i m}(t) & =x_{2}(t)
\end{aligned}
$$

Due to the related adjacency matrices (Eq. 12) a digraph (Fig. 1) can be generated. Obviously, a suitable flat input $u^{\text {flat }}(t)$ should enter at node $x_{3}$ to provide the most distantly shortest path.

$$
A^{*}=\left[\begin{array}{lll}
0 & 0 & 1 \\
1 & 0 & 0 \\
0 & 1 & 0
\end{array}\right] ; \quad C^{*}=\left[\begin{array}{lll}
0 & 1 & 0
\end{array}\right]
$$

According to the above outcome, a potential input-affine DDE system (Eq. 13) can be derived.

$$
\begin{aligned}
\dot{x}_{1}(t) & =x_{3}\left(t-\tau_{1}\right) \\
\dot{x}_{2}(t) & =x_{1}(t) \\
\dot{x}_{3}(t) & =x_{2}(t)+u^{\text {flat }}(t) \\
y^{\text {sim }}(t) & =x_{2}(t)
\end{aligned}
$$

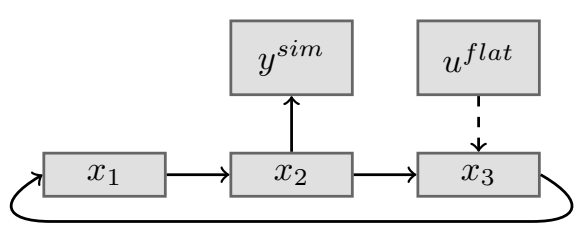

Figure 1. Digraph of the illustrative example. For a given output $y^{\text {sim }}$, the flat input $u^{\text {flat }}$ has to act on state $x_{3}$ to provide a maximum distance to get from $u^{\text {flat }}$ to $y^{\text {sim }}$ using a minimum number of edges.

In this case, the DDE system (Eq. 13) is indeed a flat system, i.e., all states $x_{i}(t)$, as well as the flat input $u^{\text {flat }}(t)$ can be recalculated by the output $y^{\text {sim }}(t)$ and derivatives thereof (Eq. 14-17).

$$
\begin{aligned}
x_{2}(t) & =y^{\text {sim }}(t) \\
x_{1}(t) & =\dot{y}^{\text {sim }}(t) \\
x_{3}\left(t-\tau_{1}\right) & =\ddot{y}^{\text {sim }}(t) \\
u^{\text {flat }}(t) & =\dddot{y}^{\text {sim }}\left(t+\tau_{1}\right)-y^{\operatorname{sim}}(t)
\end{aligned}
$$

Generally, more complex systems can be checked in this way by highly efficient methods of graph analysis, e.g., the algorithm of Shortest Path [Dijkstra (1959)] can be applied.

\subsection{Global Sensitivity Analysis}

Especially for models in systems biology, the influence of model parameters $\theta$ on the model output varies strongly. On the one hand there are parameters $\theta_{l} \subset \theta$ that can be changed by orders of magnitude without notable influence on the dynamic behavior and on the other hand a slight change of certain parameters $\theta_{h} \subset \theta$ leads to a strong output variation. Evidently, in the framework of parameter identification it is much more easier to identify the latter subset $\theta_{h}$.

To determine the influence of model parameters $\theta$ on $y^{\text {sim }}(t)$ related parameter sensitivities have to be calculated. If identified parameters $\theta$ provide tight confidence regions, i.e., their values are almost certainly known, then the sensitivities can be determined by a local method using the sensitivity matrix (SM) (Eq. 18).

$$
S M\left(t_{k}\right)=\left.\frac{\partial y^{s i m}\left(t_{k}\right)}{\partial \theta}\right|_{\theta}
$$

Usually, this is not the case and global methods taking parameter uncertainties explicitly into account have to be applied. These requirements are automatically fulfilled by variance-based approaches. Treating parameters $\theta$ and the output $y^{\operatorname{sim}}(t)$ as random variables, the amount of variance that each parameter $\theta_{i}$ contributes to the variance of the output $\sigma^{2}\left(y^{\text {sim }}(t)\right)$ is determined.

The ranking of a parameter $\theta_{i}$ is done by the amount of output variance that would vanish, if this parameter $\theta_{i}$ is assumed to be known. Formally, for every assumed known parameter $\theta_{i}$ a conditional variance $\sigma_{-i}^{2}\left(y^{\text {sim }} \mid \theta_{i}\right)$ can be determined. The subscript $-i$ indicates that the variance is taken over all parameters other than $\theta_{i}$. As $\theta_{i}$ itself is a random variable in reality, the expected 
value of the conditional variance $\underset{i}{E}\left[{ }_{-i}^{\sigma^{2}}\left(y^{s i m} \mid \theta_{i}\right)\right]$ has to
be determined, where the subscript $E$ indicates that the be determined, where the subscript $\underset{i}{E}$ indicates that the expected value is only taken over the parameter $\theta_{i}$. Now, the output variance $\sigma^{2}\left(y^{\text {sim }}\right)$ can be separated [Saltelli et al. (2005)] into the following two additive terms.

$$
\left.\sigma^{2}\left(y^{s i m}\right)=\underset{i}{\sigma_{i}^{2}} \underset{-i}{E}\left[y^{s i m} \mid \theta_{i}\right]\right)+\underset{i}{E}\left[{\underset{-i}{\sigma}}^{2}\left(y^{s i m} \mid \theta_{i}\right)\right]
$$

The variance of the conditional expectation $\sigma_{i}^{2}\left(\underset{-i}{E}\left[y^{\operatorname{sim}} \mid \theta_{i}\right]\right)$ represents the contribution of parameter $\theta_{i}$ to the variance $\sigma^{2}\left(y^{s i m}\right)$ indicating the importance of this parameter. The normalized expression (Eq. 20) is known as the first order sensitivity index [Sobol' (1993)] and shall be used in the following for parameter sensitivity analysis.

$$
S_{i}^{y}=\frac{\sigma_{i}^{2}\left(\underset{-i}{E}\left[y^{\text {sim }} \mid \theta_{i}\right]\right)}{\sigma^{2}\left(y^{\text {sim }}\right)}
$$

Analogous considerations can be made for flat inputs $u^{f l a t}(t)$. Equivalent counterparts of first order indices are given in (Eq. 21). As shown in section 3, insensitive parameters related to outputs $y^{\text {sim }}(t)$ do not have to be insensitive to $u^{\text {flat }}(t)$. This interesting feature enables a novel perspective on optimal experimental design (OED) for parameter identification. In general, OED attempts to obtain sensitive parameters by optimally designed operation conditions [Kreutz and Timmer (2009); Heine et al. (2008); Schenkendorf et al. (2009)], i.e., new costly experiments have to be conducted in this case. By evaluating cost functions (Eq. 6-10) that are based on flat inputs a new spectrum of parameter sensitivities is provided. Naturally, there is no guarantee that these new parameter sensitivities are more suitable for parameter identification but they are worth to be checked a-priori before running any additional experiment.

$$
S_{i}^{u}=\frac{\sigma_{i}^{2}\left(\underset{-i}{E}\left[u^{\text {flat }} \mid \theta_{i}\right]\right)}{\sigma^{2}\left(u^{\text {flat }}\right)}
$$

Usually, the integrals $\left(\sigma^{2}\left(y^{\text {sim }}\right)\right.$ or $\left.\sigma^{2}\left(y^{\text {sim }} \mid \theta_{i}\right)\right)$ are evaluated by Monte Carlo methods. This is correlated with a high computational effort. To reduce computation costs and to avoid a random exploration of the parameter space $\mathbb{R}^{p}$ by using Monte Carlo methods [Sobol' (2001)] the unscented transformation is used instead [Schenkendorf and Mangold (2011) and references therein].

\section{APPLICATION}

The following DDE system (Eq. 22) describes the influenza A virus production in large-scale microcarrier culture by a model similar to [Möhler et al. (2005)]. Uninfected MadinDarby canine kidney (MDCK) cells $U_{c}(t)$ are infected by active viruses $V_{i}(t)$. After a certain delay time of $\tau_{1}$ infected MDCK cells $I_{c}(t)$ release active and inactive virus particles $V_{i}(t)$ and $V_{d}(t)$, respectively. The active virus particles either infect the remaining uninfected cells or are degraded to inactive virions.

$$
\begin{aligned}
& \frac{d U_{c}(t)}{d t}= \theta_{6} \frac{C_{\max }-\left(U_{c}(t)+I_{c}(t)\right)}{C_{\max }} U_{c}(t)- \\
& \theta_{1} U_{c}(t) V_{i}(t) \\
& \frac{d I_{c}(t)}{d t}=\theta_{1} U_{c}(t) V_{i}(t)-\theta_{2} I_{c}(t) \\
& \frac{d V_{i}(t)}{d t}=\theta_{3} I_{c}\left(t-\tau_{1}\right)-\theta_{4} V_{i}(t)-\theta_{1} U_{c}(t) V_{i}(t) \\
& \frac{d V_{d}(t)}{d t}=\theta_{5} I_{c}\left(t-\tau_{1}\right)+\theta_{4} V_{i}(t) \\
& \\
& y_{1}(t)=V_{i}(t) \\
& y_{2}(t)=V_{d}(t)
\end{aligned}
$$

In practice, the concentrations of active and inactive virus particles are measurable (Eq. 23-24). With these measurement quantities and Eq. 22 the related digraph (Fig. 2) can be derived. As two quantities are measured two flat inputs, $u_{1}^{\text {flat }}(t)$ and $u_{2}^{\text {flat }}(t)$, have to be determined. Due to the distance criteria (Sec. 2.2), as rule of thumb, these inputs should act on $U_{c}(t)$ and $I_{c}(t)$ (Eq. 25), respectively.

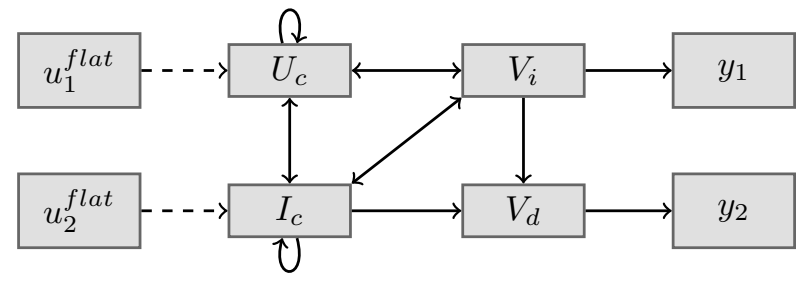

Figure 2. Digraph of the influenza A virus production model (Eq. 22). Here, the two potential flat inputs $u_{1}^{\text {flat }}$ and $u_{2}^{\text {flat }}$ should act on the states $U_{c}$ and $I_{c}$ to fulfill the distance criteria, a rule of thumb.

$$
\begin{aligned}
\frac{d U_{c}(t)}{d t}= & \theta_{6} \frac{C_{\max }-\left(U_{c}(t)+I_{c}(t)\right)}{C_{\max }} U_{c}(t)- \\
& \theta_{1} U_{c}(t) V_{i}(t)+u_{1}^{f l a t}(t) \\
\frac{d I_{c}(t)}{d t}= & \theta_{1} U_{c}(t) V_{i}(t)-\theta_{2} I_{c}(t)+u_{2}^{f l a t}(t) \\
\frac{d V_{i}(t)}{d t}= & \theta_{3} I_{c}\left(t-\tau_{1}\right)-\theta_{4} V_{i}(t)-\theta_{1} U_{c}(t) V_{i}(t) \\
\frac{d V_{d}(t)}{d t}= & \theta_{5} I_{c}\left(t-\tau_{1}\right)+\theta_{4} V_{i}(t)
\end{aligned}
$$

As states and inputs (Eq. 26-30) can be expressed explicitly by the outputs (Eq. 23-24) and derivatives thereof, the DDE system (Eq. 25) is differentially flat.

$$
\begin{aligned}
I_{c}\left(t-\tau_{1}\right)= & \frac{1}{\theta_{5}}\left(\dot{y}_{2}(t)-\theta_{4} y_{1}(t)\right) \\
U_{c}(t)= & \frac{\theta_{3} I_{c}(t-\tau)-\theta_{4} y_{1}(t)-\dot{y}_{1}(t)}{\theta_{1} y_{1}(t)} \\
u_{2}^{\text {flat }}(t)= & \frac{1}{\theta_{5}}\left(\ddot{y}_{2}\left(t+\tau_{1}\right)-\theta_{4} \dot{y}_{1}\left(t+\tau_{1}\right)\right)- \\
& \theta_{1} U_{c}(t) y_{1}(t)+\theta_{2} I_{c}(t)
\end{aligned}
$$




$$
\begin{aligned}
u_{1}^{f l a t}(t)= & \mu\left(t+\tau_{1}\right) U_{c}\left(t+\tau_{1}\right)+\theta_{1} U_{c}\left(t+\tau_{1}\right) y_{1}\left(t+\tau_{1}\right)- \\
& \frac{\theta_{4} \dot{y}_{1}\left(t+\tau_{1}\right)-\ddot{y}_{1}\left(t+\tau_{1}\right)}{\theta_{1} y_{1}\left(t+\tau_{1}\right)}- \\
& \frac{\theta_{3}\left(\theta_{1} U_{c}(t) y_{1}(t)-\theta_{2} I_{c}(t)+u_{f_{2}}(t)\right)}{\theta_{1} y_{1}\left(t+\tau_{1}\right)}- \\
& \frac{U_{c}\left(t+\tau_{1}\right) \dot{y}_{1}\left(t+\tau_{1}\right)}{y_{1}\left(t+\tau_{1}\right)}
\end{aligned}
$$

with

$\mu\left(t+\tau_{1}\right)=\theta_{6} \frac{C_{\max }-\left(U_{c}\left(t+\tau_{1}\right)+I_{c}\left(t+\tau_{1}\right)\right)}{C_{\max }}$

After the successful transformation to a flat system, the proposed method of parameter identification can be applied. The cultivation of MDCK cells is done in a batch mode, i.e., the generated two flat inputs (Eq. 25) are just fictitious. Consequently, the cost function (Eq. 10) has to be evaluated for the identification of model parameters $\theta$ and the delay parameter $\tau_{1}$, respectively.

Assuming almost perfect measurement data, i.e., high sample rate without measurement noise, the model parameters $\theta$ are estimated for 100 different delay parameter values equally spaced in the the range of 5 to 15 hours, $\tau_{1} \in[5,15]$ h. In figure (Fig. 3), the cost function (Eq. 10) has a global minimum at $\tau_{1}=7.5 \mathrm{~h}$ which is the correct result. The estimated model parameters $\theta$ at this optimally determined delay parameter are given in Tab. 1. Although the initial parameter values $\theta_{I n i}$ are far away from the true parameter values $\theta_{\text {True }}$, the proposed optimization framework is able to provide reliable estimates $\theta_{O p t}$ in a feasible computational time. The overall cpu-time is less than 10 seconds in this case. In comparison to the evaluation of the standard cost function (Eq. 4) it is significant speedup.

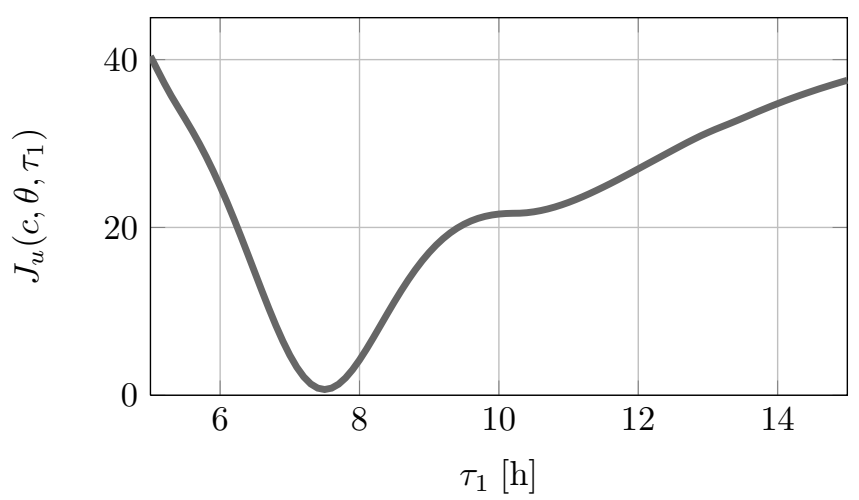

Figure 3. The novel cost function $J_{u}\left(c, \theta, \tau_{1}\right)$ (Eq. 10) evaluated at different time-delay parameter values $\tau_{1}$. In detail, an optimizer is initialized iteratively at 100 different $\tau_{1}$ values, $\tau_{1} \in[5,15] \mathrm{h}$. The overall cpu-time is less than 10 seconds in this case.

In the framework of parameter identification also robustness against initial parameter values, $\theta^{I n i}$ and $\tau_{1}^{I n i}$, is a crucial factor. Here, the standard approach (Eq. 4) is much more sensitive to initial parameter values than the flat input approach. For instance, only under the ideal condition of $\tau_{1}=7.5 \mathrm{~h}$ the model parameters $\theta$ are identified properly. A slight deviation from $\tau_{1}$ leads to a divergence of the optimization routine (Fig. 4). Finally,

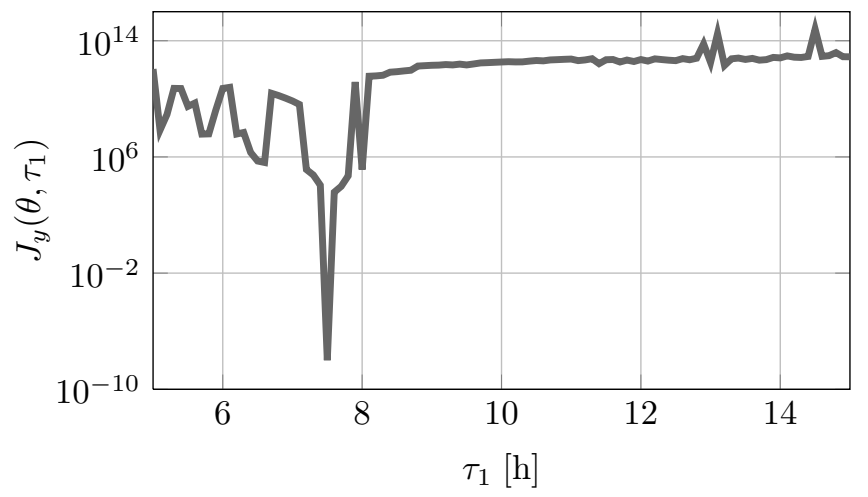

Figure 4. The standard cost function $J_{y}\left(\theta, \tau_{1}\right)$ (Eq. 4) evaluated at different time-delay parameter values $\tau_{1}$. In detail, an optimizer is initialized iteratively at 100 different $\tau_{1}$ values, $\tau_{1} \in[5,15] \mathrm{h}$. A slight deviation of the true $\tau_{1}$ value, $\tau_{1}=7.5 \mathrm{~h}$, leads to a divergence of the optimization routine.

the change in parameter sensitivities is demonstrated for the differentially flat DDE system (Eq. 25). In the following, a relative parameter perturbation of $25 \%$ and fixed operation conditions are assumed. The corresponding parameter sensitivity spectrum of the standard approach (Eq. 20) indicates that model parameter $\theta_{4}$ is the least sensitive one (Fig. 5(a)). Here, as previously suggested, the parameter sensitivities are investigated by the novel approach described above(Eq. 21). As shown (Fig. 5(b)), the spectrum of parameter sensitivities is changed significantly. The previously insensitive model parameter $\theta_{4}$ is now the most sensitive one, i.e., parameter $\theta_{4}$ is likely to be identified properly. This result agrees well with the previous outcome of the actual parameter identification (Tab. 1), i.e., the model parameter $\theta_{4}$ is reconstructed best. In particular, the existing measurement data set is used just in a different way (Eq. 10). There is no need of OED and any additional experiment to improve the sensitivity of $\theta_{4}$.

\section{CONCLUSION}

We have introduced and analyzed the concept of flat inputs in field of parameter identification of DDE models. Here, a differentially flat DDE system is transformed to an algebraic input/output representation that obviates the need for numerical integration of the DDEs. This strategy leads to a significant speedup in the parameter identification part.

In a first step, potential flat inputs $u^{\text {flat }}(t)$ of a mathematical model of the influenza $A$ virus production are determined by a rule of thumb and verified subsequently. All states $x(t)$ and flat inputs $u^{f l a t}(t)$ are expressed by given outputs $y^{\operatorname{sim}}(t)$ and derivatives thereof. Now, these flat inputs are used to define a novel cost function for parameter identification.

Beyond that, the presented approach has a number of merits. In addition to the significant reduction in computational time, the cost function based on $u^{\text {flat }}(t)$ are robust against initial values of model parameters $\theta$ and 


\begin{tabular}{|c|cccccc|}
\hline & $\theta_{1}$ & $\theta_{2}$ & $\theta_{3}$ & $\theta_{4}$ & $\theta_{5}$ & $\theta_{6}$ \\
\hline$\theta_{\text {Ini }} / \theta_{\text {True }}$ & 750 & 750 & 750 & 750 & 750 & 750 \\
$\theta_{\text {Opt }} / \theta_{\text {True }}$ & 1.0032 & 1.0014 & 0.9974 & 1.000 & 0.9974 & 0.9976 \\
\hline
\end{tabular}

Table 1. Result of parameter identification by minimizing a cost function based on flat inputs $u_{\text {flat }}(t)$ (Eq. 10). Despite the initial parameter deviation $\theta_{\text {Ini }}$, the identified model parameters $\theta_{O p t}$ are close to the true values $\theta_{\text {True }}$.

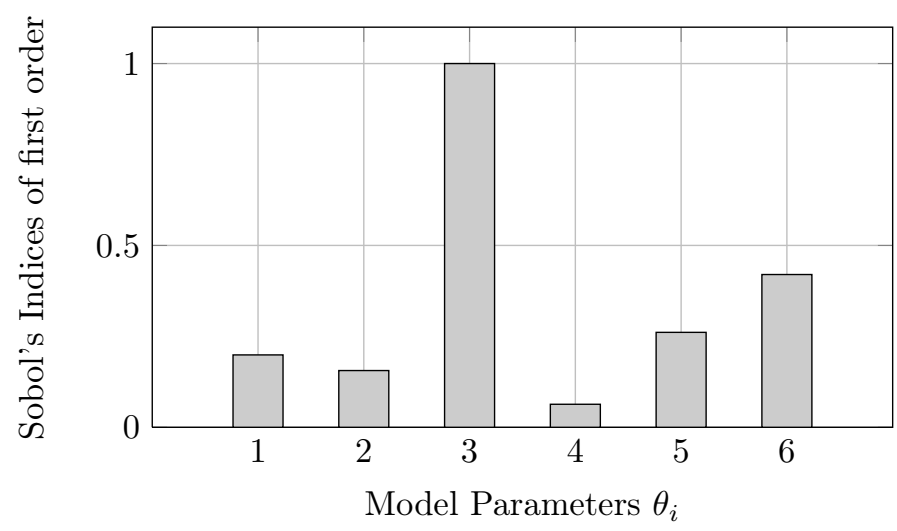

(a) Standard approach (Eq. 20)

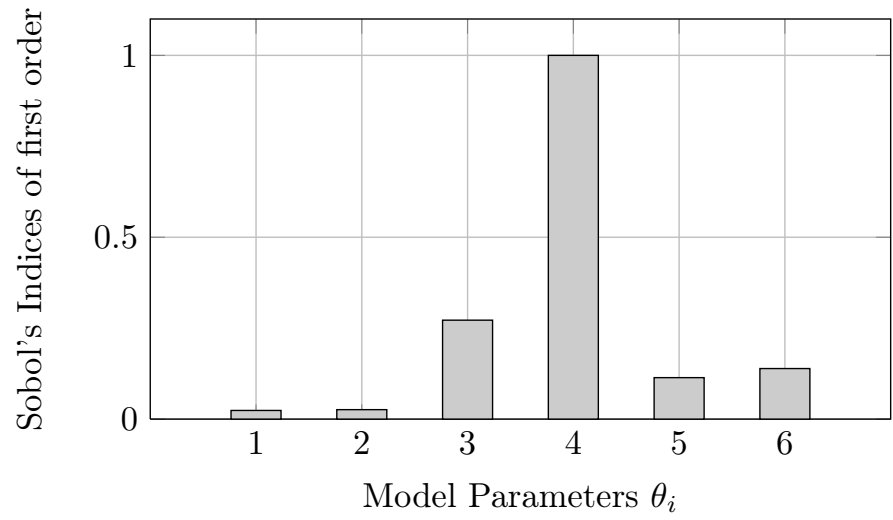

(b) Flat input approach (Eq. 21)

Figure 5. Integral measures of Sobol's indices $\int_{0}^{T} S_{i} d t$ normalized to the most sensitive parameter are presented for two different strategies of parameter identification. In the upper case, the spectrum of parameter sensitivities is shown for the standard approach, i.e., parameter sensitivities related to outputs $y^{\text {sim }}(t)$. Below, parameter sensitivities related to flat inputs $u^{\text {flat }}(t)$ are given. Using the same measurement data $y^{\text {data }}\left(t_{k}\right)$, there is a significant change in the range of sensitivities.

$\tau_{1}$, respectively. Also a change in parameter sensitivities is addressed that opens a new perspective in optimal experimental design for parameter identification.

The sensitivity of the presented approach to measurement noise is the subject of ongoing research.

\section{REFERENCES}

Dijkstra, E.W. (1959). A note on two problems in connexion with graphs. Numerische Mathematik, 269-271.
Graichen, K., Hagenmeyer, V., and Zeitz, M. (2005). A new approach for inversion-based feedforward control design of non-linear systems. Trans. Autom. Control, 41, 2033-2041.

Heine, T., Kawohl, M., and King, R. (2008). Derivativefree optimal experimental design. Chemical Engineering Science, 63, 4873-4880.

Kreutz, C. and Timmer, J. (2009). Systems biology: experimental design. FEBS Journal, 276, 923-942.

Lévine, J. (2009). Analysis and Control of Nonlinear Systems - A Flatness-based Approach. Springer.

Möhler, L., Flockerzi, D., Sann, H., and Reichl, U. (2005). Mathematical model of influenza a virus production in large-scale microcarrier culture. Biotechnology and Bioengineering, 90, 46-58.

Poyton, A.A., Varziri, M.S., McAuley, K.B., McLellan, P.J., and Ramsay, J.O. (2006). Parameter estimation in continuous-time dynamic models using principle differential analysis. Computers and Chemical Engineering, 30, 698-708.

Ramsay, J.O. and Silverman, B.W. (2005). Functional Data Analysis. Springer New York.

Saltelli, A., Ratto, M., Tarantola, S., and Campolongo, F. (2005). Sensititivity analysis for chemical models. Chemical Reviews, 105, 2811âĂŞ2828.

Schenkendorf, R., Kremling, A., and Mangold, M. (2009). Optimal experimental design with the sigma point method. IET Systems Biology, 3, 10-23.

Schenkendorf, R. and Mangold, M. (2011). Qualitative and quantitative optimal experimental design for parameter identification. In 18th IFAC World Congress Milano (Italy).

Sira-Ramirez, H. and Agrawal, S.K. (2004). Differentially Flat Systems. Marcel Decker, Inc.

Sobol', I.M. (1993). Sensititivity analysis for nonlinear mathematical models. Mathematical Modeling and Computational Experiment, 1, 407-414.

Sobol', I.M. (2001). Global sensitivity indices for nonlinear mathematical models and the monte carlo estimates. Ma, 55, 271-280.

Varziri, M.S., Pyoton, A.A., McAuley, K.B., McLellan, P.J., and Ramsay, J.O. (2008). Selecting optimal weighting factors in ipda for parameter estimation in continuous-time dynamic models. Computers and Chemical Engineering, 32, 3011-3022.

Waldherr, S. and Zeitz, M. (2008). Conditions for the existence of a flat input. International Journal of Control, 81, 439-443.

Waldherr, S. and Zeitz, M. (2010). Flat inputs in the mimo case. In IFAC Symposium on Nonlinear Control Systems.

Wey, T. (2002). Nichtlineare Regelungssysteme - Ein differentialalgebraischer Ansatz. Teubner. 